\title{
Open magnetic flux tubes in the corona and the transport of solar energetic particles
}

\author{
K.-L. Klein ${ }^{1}$, S. Krucker ${ }^{2}$, G. Lointier ${ }^{1, \star}$, and A. Kerdraon ${ }^{1}$ \\ 1 Observatoire de Paris, LESIA-CNRS UMR 8109, 92195 Meudon, France \\ e-mail: ludwig.klein@obspm.fr \\ 2 Space Sciences Laboratory, University of California, Berkeley, CA 94720-7450, USA \\ e-mail: krucker@ssl.berkeley.edu
}

Received 11 December 2007 / Accepted 6 May 2008

\begin{abstract}
We investigate how magnetic fields guide energetic particles through the corona into interplanetary space and eventually to a spacecraft near the Earth. A set of seven simple particle events is studied, where energetic electrons (30-500 keV; Wind spacecraft) or protons (5-55 MeV; SoHO) were released together with low-energy electron beams producing metric-to-kilometric type III emission. Imaging of the coronal (metre-wave) part of this emission with the Nançay Radioheliograph is used to identify the open flux tubes that guide these electrons - and by inference all particles detected at $1 \mathrm{AU}$. Open coronal field lines are also computed using potential magnetic field extrapolations, constrained by a source surface and by SoHO/MDI measurements in the photosphere (code by Schrijver and DeRosa). We find that in all events the type III radio sources lie in open flux tubes in the potential magnetic field extrapolations. The open flux tubes are rooted in small parts of the parent active region, covering a heliocentric angle of a few degrees in the photosphere. But they expand rapidly above the neighbouring closed magnetic structures and cover several tens of degrees in longitude on the source surface. Some of these open field lines are found to connect the parent active region to the footpoint of the nominal Parker spiral on the source surface, within the uncertainty of about $\pm 10^{\circ}$ inherent to the evaluation of its connection longitude. This is so even when the parent active region is as far as $50^{\circ}$ away. In two cases where the coronal flux tubes point to high heliolatitudes, the detection of Langmuir waves at the Wind spacecraft in the ecliptic plane suggests that the interplanetary field lines curve down to the ecliptic before reaching $1 \mathrm{AU}$. We conclude that non-radial open flux tubes in the corona can transport particles over several tens of degrees in longitude even in simple impulsive particle events. In all events we studied, potential magnetic field models give an adequate description of these structures.
\end{abstract}

Key words. Sun: particle emission - Sun: corona - Sun: radio radiation - Sun: magnetic fields - Sun: flares Sun: solar-terrestrial relations

\section{Introduction}

Enhanced fluxes of solar energetic particles (SEP) - so-called SEP events - accompany processes of explosive energy conversion in the corona, notably flares and coronal mass ejections (CMEs). From Parker's model of the interplanetary magnetic field, it is expected that particles accelerated in the corona reach the Earth, provided they are injected into interplanetary magnetic flux tubes near $50^{\circ}-60^{\circ}$ western longitude. Statistically speaking, SEP events conform to this picture: their associated solar activity occurs most often in the western hemisphere, and higher peak fluxes are usually measured by spacecraft near the nominally well-connected field line than at remote places (see reviews by Kunow et al. 1991; Reames 1999). But there is a large scatter. For example, Lin (1970) and Kallenrode et al. (1992) showed that electrons in flare-associated - so-called "impulsive" - SEP events can come from flares as far as $50^{\circ}$ away in longitude from the nominal Parker spiral connection. Nitta et al. (2006) found a similar result for a large set of ${ }^{3} \mathrm{He}$-rich SEP events at $\mathrm{MeV} /$ nuc-energies. Reinhard \& Wibberenz (1974) showed that the rise time of $\sim 10 \mathrm{MeV}$ proton fluxes to maximum is independent of the longitude of the parent flare within a broad longitude range from $\mathrm{W} 10^{\circ}$ to $\mathrm{W} 100^{\circ}$ (see also Fan et al. 1968;

* Now at Laboratoire de Physique et Chimie de l'Environnement, 3 A, ave de la Recherche Scientifique, 45071 Orléans Cedex 2, France. van Hollebeke et al. 1975). They called this longitude range "fast propagation region". Wibberenz \& Cane (2006) showed that for relativistic electron events this region may comprise flares up to $90^{\circ}$ from the well-connected field line.

How is it possible that SEP from a broad range of longitudes have direct access to a given spacecraft, rather than only those particles which are accelerated close to the nominal Parker spiral? Shock-wave acceleration in a broad cone of open magnetic field lines is often offered as an explanation (Carmichael 1962; Lin 1970; Reames 1999). But EUV or X-ray images of the corona also show that the magnetic field lines in the low corona are complex. They might therefore provide longitudinal transport of particles from localised acceleration sites in an active region to the remote footpoint of the interplanetary field line connected with the spacecraft. Early work on transport guided by coronal magnetic fields is reviewed in Perez-Peraza (1986) and Kunow et al. (1991). At that time two types of situations were discussed: broad cones of open field lines extending down to the photosphere or complex particle motions in closed magnetic structures and drifts to escape from them. Here we address the question to which extent and over which longitude ranges open magnetic flux tubes transport charged particles accelerated in flaring active regions.

A number of authors have recently compared open coronal magnetic field lines rooted in active regions, as inferred from 
the current-free (potential) extrapolation of photospheric magnetic field measurements, with the presumed solar sources of impulsive SEP events (Wang et al. 2006; Pick et al. 2006; Nitta et al. 2006). We study a set of carefully identified events where the release of near-relativistic electrons (30-500 keV) and deka$\mathrm{MeV}$ protons (5-55 MeV) to interplanetary space occurred together with the production of electron beams whose radio emission ("type III bursts") could be traced from the corona (at metre waves) to $1 \mathrm{AU}$ (kilometre waves). We use both potential magnetic field modelling and radio snapshot maps of the type III sources in the corona, which provide an alternative means to identify open magnetic flux tubes and to localise the parent active region of the SEP events. In Sect. 2 we present the two complementary techniques and demonstrate that they provide consistent identifications of the open flux tubes which guide particles through the corona. We discuss the evolution of these flux tubes with height and their connection with the interplanetary magnetic field in longitude and latitude. Preliminary results of the present study were reported by Lointier et al. (2004).

\section{Observations and analysis}

\subsection{Radio identification of magnetic flux tubes in the corona}

The present work starts from two samples of 18 near-relativistic electron events (energies above $30 \mathrm{keV}$; Table 1 in Klein et al. 2005) and of six impulsive deka-MeV proton events (energies (5-55) MeV; Klein \& Posner 2005) detected at the Wind and SoHO spacecraft, respectively. The energy-dependent arrival at the spacecraft of the first detected particles was used in these publications to determine the solar release time to within about 5 to $10 \mathrm{~min}$. We analysed those events where one or several decametric type III bursts were observed in the initial release window by the WAVES experiment aboard the Wind spacecraft (Bougeret et al. 1995) or by the Nançay Decametric Array (Lecacheux 2000), and which were accompanied by metre wave emission mapped by the Nançay Radioheliograph (NRH in the following; Kerdraon \& Delouis 1997). The type III bursts show when electrons were released to interplanetary space. The common timing, within the uncertainty of the method of release time determination, suggests that the type III emitting electron beams (typical energy $10 \mathrm{keV}$, e.g. Buttighoffer 1998) and at least the first near-relativistic electrons and deka-MeV protons detected in situ were accelerated together and were guided through the corona by the same magnetic flux tubes.

The NRH maps at frequencies between 150 and $432 \mathrm{MHz}$ with a spatial resolution of $\sim 3^{\prime}$ at $164 \mathrm{MHz}$ allow us to localise the flux tubes at heliocentric distances between 1.1 and $1.5 R_{\odot}$, provided the metre wave emission is the high-frequency extension of the decametric type III burst. In order to make sure that this is the case, we examined dynamic spectrograms observed by the radio spectrographs of the Potsdam Astrophysical Institute at Tremsdorf (Mann et al. 1992, henceforth OSRA), the University of Athens (ARTEMIS, Caroubalos et al. 2001), and IZMIRAN near Moscow ${ }^{1}$. The metre wave spectra displayed numerous densely packed bursts. Upon closer inspection of the spectra of OSRA or ARTEMIS, many of these bursts turned out to be of limited bandwidth or to resemble broadband pulsations rather than type III bursts. For the present study we retained only those events where type III bursts were seen in dynamic spectra with $1 \mathrm{~s}$ resolution or better between at least one NRH frequency (i.e.

\footnotetext{
1 http://www.aip.de/groups/osra/, http://www.cc.uoa.gr/ artemis/, http://helios.izmiran.rssi .ru/lars/LARS .html
}

at least $164 \mathrm{MHz}$ ) and the low-frequency limit of the spectrograph. We identified six SEP events from Klein et al. (2005) and Klein \& Posner (2005) which satisfied the above criteria. One event (2003 Oct. 04) was added because the solar release time of near relativistic electrons could be determined with high accuracy from measurements of the Three-dimensional Plasma and Energetic Particles Instrument on the Wind spacecraft (henceforth Wind/3DP; Lin et al. 1995).

\subsection{Computation of open magnetic field lines in the corona and interplanetary space}

SEP events such as those discussed here are in general not supposed to be accelerated at CME shocks. Klein \& Posner (2005) showed specifically that although their impulsive proton events were accompanied by fast and narrow CMEs, the first protons arriving at $1 \mathrm{AU}$ and the radio emitting electron beams were accelerated in the corona after the CME passage, not at its bow shock. Since the SoHO/LASCO movies ${ }^{2}$ of these CMEs suggest that they propagate along pre-existing field lines without substantially modifying them, we assume that the magnetic field configuration which guides the energetic particles through the corona is quasi-static.

In a quasi-static situation coronal magnetic field lines can be traced by extrapolating photospheric field measurements. It is common to use a current free (potential) field model to identify large-scale coronal structures. While such models are clearly insufficient to represent the sheared and twisted magnetic field configurations in active regions, they have been successful in connecting coronal and interplanetary structures on larger scales. For example, Schrijver \& DeRosa (2003) and Wang \& Sheeley (2003) used such models to identify the origin of the heliospheric magnetic field, while Wang et al. (2006) showed that they represent the large-scale open magnetic flux tubes which guide SEP through the corona. In these models the transition between the coronal and the interplanetary magnetic field is supposed to take place at a spherical source surface, outside of which the magnetic field is frozen into the radially flowing solar wind. Such models are designated as potential field-source surface models (PFSS models). More detailed descriptions, references to older literature, and discussions can be found in Schrijver \& DeRosa (2003), Wang et al. (2006) and Nitta et al. (2006).

Like Nitta et al. (2006), we use the PFSS code of Schrijver \& DeRosa (2003) which is available in the idl-based SolarSoft data analysis package ${ }^{3}$. The extrapolation is based on evolving synoptic maps assembled from measurements of the lineof-sight component of the magnetic field in the photosphere by the Michelson Doppler imager (MDI) aboard the Solar and Heliospheric Observatory (SoHO; Scherrer et al. 1995), assuming the magnetic field to be orthogonal to the photosphere. The model is updated by directly inserting MDI magnetogram data as it becomes available. After flux rotates out of the assimilation window (which extends out to about $60^{\circ}$ from disc centre), it continues to evolve subject to transport processes based on the observed effects of differential rotation, meridional flows, convective dispersal, and the merging and fragmentation of flux. This model has been successfully applied to analyse the observed distribution of photospheric flux (Schrijver 2001) and aspects of its long-term dispersal across the surface (Schrijver \& Title 2001). We then suppose that the SEP reach the spacecraft

\footnotetext{
2 http://lasco-www.nrl.navy.mil/

3 pfss package, cf. http://www. lmsal. com/ derosa/pfsspack/
} 
Table 1. SEP event sample: solar wind parameters, associated activity, magnetic connection to the spacecraft.

\begin{tabular}{|c|c|c|c|c|c|c|c|}
\hline Date & $\begin{array}{l}\text { Time } \\
\text { (UT) } \\
\text { (2) }\end{array}$ & DOY & $\begin{array}{c}\text { SW speed } \\
{\left[\mathrm{km} \mathrm{s}^{-1}\right]} \\
(4)\end{array}$ & $\begin{array}{l}\text { Longitude } \\
\text { (Parker) } \\
\text { (5) }\end{array}$ & $\begin{array}{l}\text { Longitude } \\
\text { (Flare) } \\
\text { (6) }\end{array}$ & $\begin{array}{c}\text { Longitude } \\
\text { (AR of III) } \\
\text { (7) }\end{array}$ & $\begin{array}{r}\text { Longit. } \\
\text { Distance } \\
\text { (8) }\end{array}$ \\
\hline 20000419 & $12: 34$ & 110.52361 & 446 & $52^{\circ}$ & $\mathrm{E}^{\circ} 4^{\circ}(8963)$ & ${\mathrm{E} 01^{\circ}(8963)}^{\prime}$ & $-53^{\circ}$ \\
\hline 20000501 & $10: 24$ & 122.43333 & 453 & $52^{\circ}$ & - & $54^{\circ}(8971)$ & $2^{\circ}$ \\
\hline 20010430 & $10: 59$ & 120.45764 & 456 & $51^{\circ}$ & - & $68^{\circ}(9433)$ & $17^{\circ}$ \\
\hline 20021005 & $12: 16$ & 278.51111 & 419 & $56^{\circ}$ & - & $64^{\circ}(10134)$ & $8^{\circ}$ \\
\hline 20021020 & $14: 20$ & 293.59722 & 666 & $35^{\circ}$ & $\begin{array}{l}18^{\circ}(10159) \\
23^{\circ}(10160) \\
25^{\circ}(10159) \\
63^{\circ}(10154)\end{array}$ & $22^{\circ}(10160)$ & $-13^{\circ}$ \\
\hline 20021212 & $12: 41$ & 346.52847 & 383 & $61^{\circ}$ & - & $36^{\circ}(10213)$ & $-25^{\circ}$ \\
\hline 20031004 & $13: 19$ & 277.55556 & 485 & $48^{\circ}$ & - & $102^{\circ}(10464)$ & $54^{\circ}$ \\
\hline
\end{tabular}

along a Parker-spiral interplanetary magnetic field line, which intersects the source surface at longitude

$\phi_{0}=\frac{\omega r}{V_{\mathrm{SW}}}\left(1-\frac{R_{\mathrm{S}}}{r}\right)$.

Here $r$ is the distance from Sun centre to the spacecraft $\left(212 R_{\odot}\right.$ for the Sun-Earth Lagrange point), $\omega$ the angular speed of solar rotation (taken here for a period of 26 days which lies between the rotation period at the equator and the Carrington rotation period of 27.3 days), $R_{S}=2.5 R_{\odot}$ the heliocentric distance beyond which the magnetic field is assumed to take on the form of the Parker spiral, and $V_{\mathrm{SW}}$ is the radial solar wind speed, assumed constant in space. Nolte \& Roelof (1973) estimated how the connection longitude is changed when a more realistic solar wind model is used, including a transition between corotation enforced by the coronal magnetic field and free expansion at heliocentric distance $r_{0} \gg R_{\mathrm{S}}$, and a radially outward increasing, rather than uniform, wind speed. The connection longitudes of the Parker spiral using $r_{0}=0.1 \mathrm{AU}$ and 0.25 AU bracket those evaluated at $R_{\mathrm{S}}$ assuming constant solar wind speed, with a range of $\pm 7^{\circ}$. Further uncertainty comes from the scattering around the nominal connection point of the Parker spiral field line. Through Monte Carlo simulations based on the level of magnetic fluctuations measured at $1 \mathrm{AU}$ in a period of small to moderate solar activity (1996-1998), Ippolito et al. (2005) estimated that the connection longitude of the Parker spiral actually scatters within a range of $\pm\left(6^{\circ}-10^{\circ}\right)$ around its nominal value. We therefore cannot expect that the nominal Parker spiral gives an estimate of the connection longitude better than, say, $\pm 10^{\circ}$. The problem of the connection latitude will be addressed separately in Sect. 2.5.

\subsection{The events}

Table 1 lists the events analysed in the following. All are near-relativistic electron events detected at Wind/3DP or SoHO/COSTEP. Deka-MeV protons were observed with SoHO/COSTEP on 2000 May 01 and in the three events of 2002. Langmuir waves were detected at the arrival of the type III emitting electrons at Wind during the events 2000 May 01, 2001 Apr. 30, 2002 Dec. 12, and 2003 Oct. 04. No Langmuir waves were detected with the 2000 Apr. 19 type III burst. On 2002 Oct. 20 Langmuir wave packets were observed, but could not be unambiguously associated with the type III burst of interest. No Wind data exist for the 2002 Oct. 05 type III burst.

Table 1 lists the event date (Col. 1), the solar release time of the energetic electrons or protons (Col. 2, with $500 \mathrm{~s}$ added for the sake of comparison with the electromagnetic emissions at Earth), and the fractional day of the year (Col. 3).
Column 4 gives the speed of the solar wind measured by Wind $/ \mathrm{SWE}^{4}$ (Ogilvie et al. 1995) for the electron events detected by Wind/3DP and by SoHO/CELIAS ${ }^{5}$ (Hovestadt et al. $1995)$ for the proton events detected by SoHO/COSTEP (2002 Oct. 05 and 20, Dec. 12). We used $1 \mathrm{~h}$ averages during the time interval containing the type III bursts. Column 5 gives the longitude of the spacecraft-connected nominal Parker spiral on the source surface. Column 6 contains the longitude of a flare $(\mathrm{H} \alpha)$ when one was reported in the Comprehensive Reports of NOAA's Solar Geophysical Data (henceforth $S G D$ ). The NOAA number of the flaring active region is given within parentheses. Column 7 gives the longitude of the active region (NOAA number in parentheses) where the footpoints of the open field lines of the PFSS extrapolation are rooted that pervade the type III radio source mapped by NRH. The coordinates were taken from $S G D$ (Sunspot groups), and interpolated to the time of the SEP release. Column 8 is the longitudinal difference between the active region (Col. 7) and the connection of the nominal Parker spiral. It is positive when the parent active region is west of the nominal Parker spiral. In several cases no flare was reported in $S G D$. The 2003 Oct. 04 flare occurred behind the limb, as shown by flare loops in the SoHO/EIT daily movie. On other occasions no $\mathrm{H} \alpha$ flare was reported during patrol hours, while a conspicuous flare appeared in the SoHO/EIT movie (e.g., 2000 May 01) .

Since these SEP events had significant electron fluxes and were associated with type III bursts from metre to kilometre waves, we refer to the particle events as "impulsive". Four of them (2000 May 01, 2002 Oct. 05, Oct. 20, Dec. 12) are also part of the sample of ${ }^{3} \mathrm{He}$-rich events studied by Wang et al. (2006).

\subsection{Type III burst mapping and open flux tubes from the PFSS model}

In Fig. 1 maps of all events of Table 1 are shown. The first and third row display the iso-intensity contours at half maximum of the selected type III bursts $(0.125 \mathrm{~s}$ snapshot maps; different colours represent different frequencies) on top of the photospheric magnetogram and the open field lines through the radio sources, computed by the PFSS model. Only the open field lines onto which the radio sources project are plotted. In the second and fourth row the same magnetic field lines are shown as they would have been seen by an observer above the Sun's north pole. The red radial line marks the longitude where the nominal Parker spiral intersects the source surface (cf. Table 1, Col. 5).

\footnotetext{
${ }^{4}$ http://web.mit.edu/afs/athena/org/s/space/www/wind. html

${ }^{5}$ http://umtof.umd.edu/pm/crn/
} 


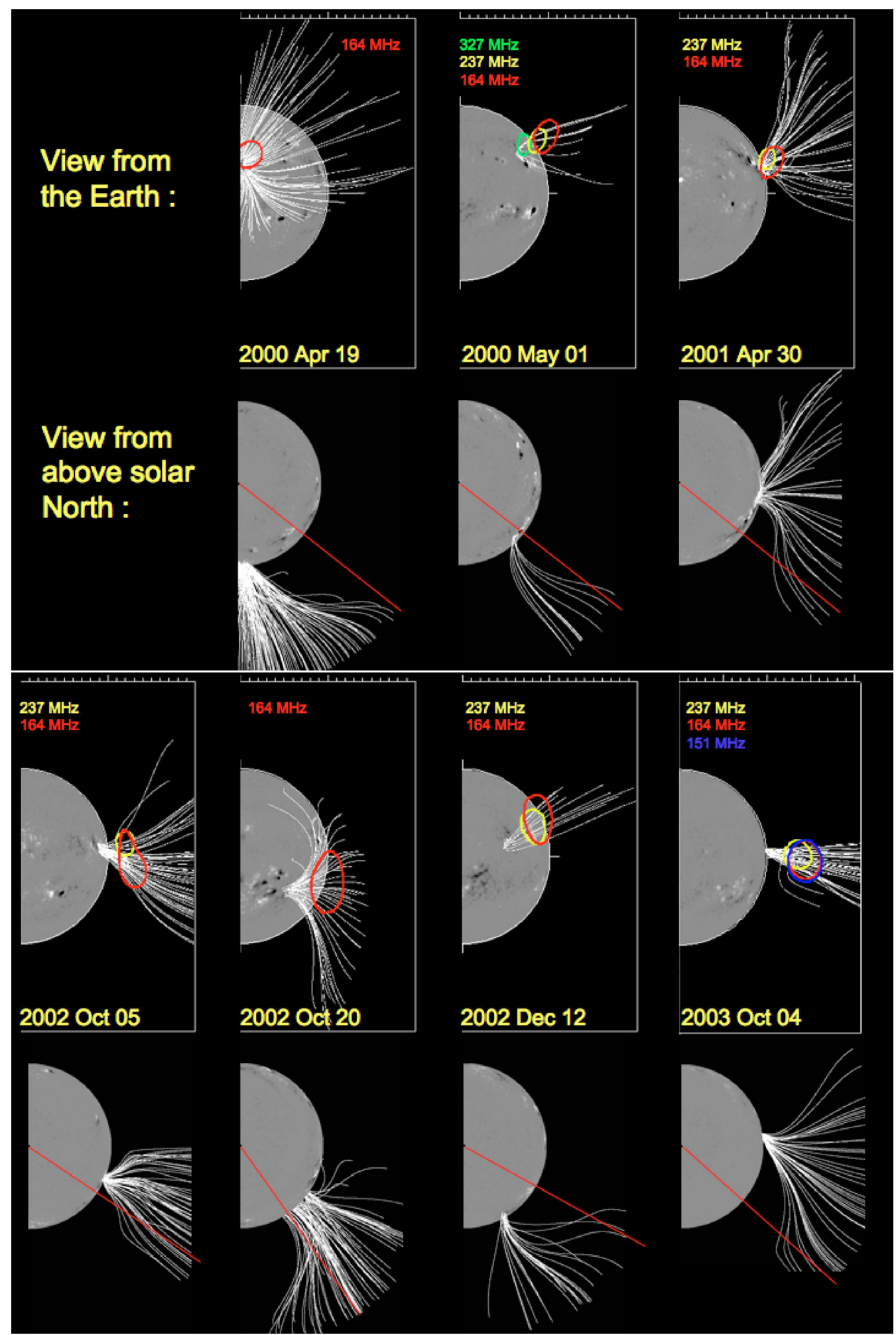

Fig. 1. Equal-intensity contours at half maximum of type III bursts (Nançay Radioheliograph; blue $151 \mathrm{MHz}$, red $164 \mathrm{MHz}$, yellow $237 \mathrm{MHz}$, green $327 \mathrm{MHz}$ ) superposed on magnetograms of the photosphere (SoHO/MDI) and open magnetic field lines computed by the PFSS model of Schrijver \& DeRosa (2003). The first and third row of maps display the view from the Earth (solar north at the top, west to the right), the second and fourth rows the view from a vantage point above the Sun's north pole (Earth toward the bottom of the figure). The red radial line marks the longitude where the nominal Parker spiral intersects the source surface.

The type III sources clearly match regions of open magnetic flux in the PFSS model. The open flux tubes are rooted in usually small monopolar areas of the photosphere. The field lines are in all cases far from a simple radial geometry, even if occasionally
(2000 May 01, 2002 Dec. 12) the view from Earth is reminiscent of such a simple picture. Most often the field lines undergo a rapid non-radial expansion in longitude, which starts at heights below $(0.5-1) R_{\odot}$ above the photosphere. E.g., the plotted 
bundle of field lines of the 2003 Oct. 04 event comprises a longitude range of hardly $4^{\circ}$ in the photosphere, $35^{\circ}$ at the height of the type III emission $\left(0.4 R_{\odot}\right)$, and $70^{\circ}$ at the source surface. Through this non-radial expansion even field lines whose photospheric root is far from the longitude of the nominal Parker spiral may connect to the Parker spiral at the source surface, to within the uncertainties of the connection longitude of $\pm 10^{\circ}$.

The non-radial expansion of the coronal flux tubes is confirmed by multi-frequency maps of the type III bursts. Radio emission near the harmonic of the local plasma frequency at 237 and $164 \mathrm{MHz}$ comes from electron densities of, respectively, $1.7 \times 10^{8}$ and $8.3 \times 10^{7} \mathrm{~cm}^{-3}$. In a hydrostatically stratified flux tube at coronal temperatures - a model that agrees with electron density measurements at eclipses for heights $\lesssim 1 R_{\odot}$ above the photosphere (Koutchmy 1994) - these levels are (0.2-0.3) $R_{\odot}$ apart. But in several events at or near the limb the NRH observes sources with little frequency dispersion of their positions:

- on 2002 Oct. 05, the $164 \mathrm{MHz}$ source has two components, while the $237 \mathrm{MHz}$ source has only one. The northern source component at $164 \mathrm{MHz}$ is exactly superposed on the $237 \mathrm{MHz}$ source.

- in the 2003 Oct. 04 type III burst the sources at 151 and $164 \mathrm{MHz}$ lie about $0.1 R_{\odot}$ above the source at $237 \mathrm{MHz}$.

This pattern can be understood if the flux tubes guiding the radio emitting electron beams are bent toward the observer so that the same, or nearly the same line of sight intercepts the radio sources at several frequencies. The view on the potential field lines from above the northern solar pole shows the geometry expected from this interpretation: field lines bending earthward from the Sun have a pronounced kink and make a small angle with the line of sight at the projected altitudes of the radio sources, at the place and with the geometry required to have similar lines of sight to the sources at 164 and $237 \mathrm{MHz}$. The situation is less clear on 2001 Apr. 30: the type III sources again suggest a flux tube which curves towards the observer and towards the nominal connection longitude on the source surface. But the polar view shows that the field lines with the adequate orientation are at higher altitude than the radio sources.

The radio maps alone do not demonstrate that the flux tubes guiding the type III emitting electrons are curved towards the observer. They might as well be curved in the opposite direction. But the fact that the type III radio emission is bright down to the vicinity of the plasma frequency at the spacecraft shows that the electron beams are guided towards the Earth, rather than away from it. In the latter case the hectometric and kilometric radio emission would be considerably weakened by directivity effects (Hoang et al. 1997). Of course, Langmuir waves would not be detected from the parent electron beams in that case. But they were detected on 2003 Oct. 04 (no Wind observations on 2002 Oct. 05).

As a further consistency check of our identification of the magnetic connection from the Sun to the spacecraft we compared the polarity of the open flux tubes in the PFSS extrapolation and the direction of the electron beams detected at Wind/3DP during all events. In the two events where the open field lines had positive polarity (2000 Apr. 19, 2001 Apr. 30) the electrons were focussed around pitch angle 0 , while in the four events with negative polarity the electrons were focussed around pitch angle $180^{\circ}$. The one-to-one correspondence of the streaming directions of the electrons at Wind with the orientation of the open field lines in the extrapolation corroborates our identification of the open coronal flux tubes which guide the particles to the spacecraft.
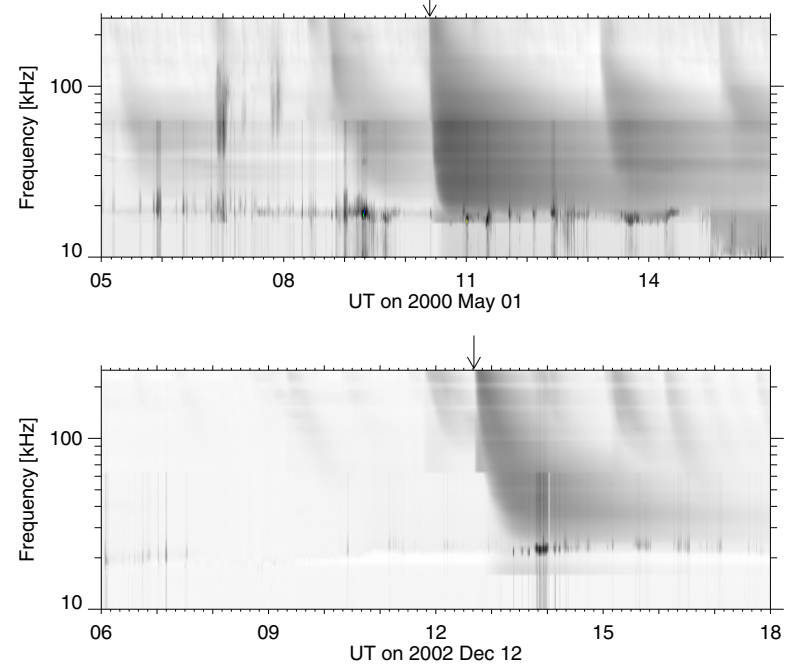

Fig. 2. Dynamic spectrograms at kilometre wavelengths from Wind/WAVES including the type III bursts on 2000 May 01 and 2002 Dec. 12. Dark shading means bright emission. The arrows above the spectrograms point to the start of the type III bursts of interest (cf. Table 1). The short-lived narrow-band emissions near $20 \mathrm{kHz}$ are Langmuir wave packets.

\subsection{Latitudinal evolution of the flux tubes in interplanetary space}

In the events on 2000 May 01 and 2002 Dec. 12 the open coronal magnetic field lines through the type III burst sources connect to the source surface at high latitudes (Fig. 1). Further from the Sun the source directions of the type III bursts at frequencies $\leq 1 \mathrm{MHz}$ can be identified using the rotational modulation of the signal received by the spinning Wind spacecraft. Measurements during the two events (courtesy S. Hoang) confirm that the centroids of the radio sources align along flux tubes which rise northward of the ecliptic plane near the Sun. Parker's model then predicts that the particles have no chance to be detected in the ecliptic near 1 AU. Nitta et al. (2006) concluded indeed that the parent active region of the 2002 Dec. 12 SEP event was not connected to the vicinity of Earth by field lines inferred from the combination of a PFSS extrapolation with the Parker model (see their Table 2). But during both events Langmuir waves were detected at the spacecraft when the type III emission approached the local electron plasma frequency. They show up as bright patches of emission at or below the low-frequency limit of the type III bursts in Fig. 2. Since Langmuir waves do not propagate away from their source region, this is significant evidence that the spacecraft intercepted the electron beams that produced the type III emission. This means that the interplanetary field lines bent down to the ecliptic between the source surface and $1 \mathrm{AU}$, in agreement with the statistical analysis of direction finding observations of type III bursts with the ISEE 3 spacecraft by Dulk et al. (1986) and with Ulysses measurements of magnetic fields and energetic particles at high heliolatitudes (see review by Neugebauer 1999).

The only way to avoid this conclusion is to ascribe the Langmuir waves to a different source than the type III emitting electron beams, and to consider the temporal association as chance coincidence. On 2000 May 01 (Fig. 2, top) Langmuir wave packets were detected repeatedly during the day, although often at the times of type III bursts, especially the one of interest in the present study. Chance coincidence cannot be ruled out. The 2002 Dec. 12 case is clearer, since a well-defined set of 
Langmuir wave packets is seen with the kilometric type III burst. We therefore conclude that the detection of Langmuir waves with the kilometric type III bursts indeed suggests that the electron beams emitting the type III bursts are seen in the ecliptic plane near $1 \mathrm{AU}$.

\section{Discussion}

\subsection{Localisation of metre wave radio sources by imaging observations}

How reliable is the identification of coronal magnetic flux tubes through radio imaging? It has often been argued that electromagnetic wave propagation in the corona, notably wave ducting, introduces appreciable shifts of the images of metre wave radio sources (Duncan 1979; McLean \& Melrose 1985; Benz 1993, Chap. 11.5). However, several observations suggest that radio maps do provide reliable localisation. For instance the sources of type III and other fast drift metric radio bursts generated by electron beams are found in well-identified open or closed largescale magnetic structures seen in white-light or soft X-ray images (Trottet et al. 1982; Aurass et al. 1994; Kundu et al. 1995; Raulin et al. 1996; Aurass \& Klein 1997; Klein et al. 1997) or in magnetic field extrapolations (Aschwanden et al. 1992). In the case of a type II burst where several harmonic lanes were observed, the source at the harmonic was found to be cospatial with that at three times the plasma frequency (Zlotnik et al. 1998). Since the effect of electromagnetic wave ducting must decrease with increasing harmonic number, wave ducting hence does not affect position measurements of harmonic plasma emission. We therefore conclude that the type III mapping does allow us to localise the magnetic flux tubes which guide the energetic particles through the corona.

\subsection{The identification of open magnetic flux tubes guiding SEP}

Our results corroborate the idea that flare-accelerated particles escape along pre-existing open magnetic flux tubes. These flux tubes have been identified in the present study with two independent and complementary techniques, PFSS modelling and radio imaging. An important element of our event selection is the simultaneity of the SEP release with type III bursts. The radio bursts demonstrate that electrons were accelerated in the corona, and allow us to identify without ambiguity the parent active region through metre wave imaging. The usefulness of this approach is illustrated by the events of 2000 Apr. 19 and 2002 Oct. 20. The 2000 Apr. 19 EIT daily movie at $19.5 \mathrm{~nm}$ wavelength shows activity in two distinct regions at the time of SEP release: AR 8963 at E04 ${ }^{\circ}$ and $\mathrm{AR} 8951$ at $\mathrm{W} 77^{\circ}$. A jet-like feature is observed in the latter region by EIT, and a narrow CME or white-light jet in the overlying corona by LASCO C2 (see the daily movies). But only AR 8963 produced metric type III bursts, and is therefore identified as the origin of the SEP. Any attempt to identify the parent active region using the SEP release timing and the activity viewed by an imager of thermal coronal emission would have been inconclusive or might have led to the identification of the wrong active region, which happened to be closer to the nominal connection longitude than the type III producing active region. On 2002 Oct. 20 several $\mathrm{H} \alpha$ flares could be associated with the SEP release. But the type III emission shows the active region where particle acceleration occurred, and the relevant open structures in the PFSS extrapolation which guided accelerated particles to interplanetary space (see Pick et al. 2006, for a similar discussion).

This illustration justifies the strict selection criteria which limit the number of events studied in the present paper. The set of complementary diagnostics that we have in these events shows that the PFSS model gives a consistent description of SEP propagation along large-scale open flux tubes in the corona, in contrast with some of the conclusions of Nitta et al. (2006). While their work is based on a ten times larger event sample than ours, it has a more ambiguous identification of the solar source region. It also evaluates the PFSS model in terms of its success in describing the latitudinal connection of the coronal magnetic field to the vicinity of the nominal Parker spiral on the source surface. But two of our events which are connected to the ecliptic plane, although the coronal magnetic flux tubes point to high heliolatitudes, seem to add further evidence to the idea that the interplanetary field may have a latitudinal component which is not described by Parker's model.

\subsection{Coronal SEP transport in open magnetic flux tubes}

It has been shown above that the non-radial expansion of open magnetic flux tubes in the corona can carry SEP over several tens of heliocentric degrees in longitude. Open field lines rooted in active regions as far as $50^{\circ}$ away from the nominal Parker spiral were found to be connected to the Parker spiral at the source surface. This magnetic connection explains the longitudinal extent of the regions with rapid access to interplanetary field lines inferred from many previous studies of SEP events (Lin 1970; Reinhard \& Wibberenz 1974; van Hollebeke et al. 1975; Kallenrode et al. 1992; Nitta et al. 2006; Wibberenz \& Cane 2006).

The result confirms an old idea, put forward, e.g., by Fan et al. (1968) and Lin (1970). But the potential field extrapolations show that there is no need to invoke large regions of open flux in the low corona or the photosphere or transport along large-scale closed loops. The open flux tubes are rooted in small parts of active regions and rapidly expand with increasing height. Their non-radial expansion is shown by the two complementary diagnostics, PFSS modelling and radio imaging. At the source surface these flux tubes cover a longitude range of several tens of heliocentric degrees. The field line bending is enforced by neighbouring closed magnetic structures in the parent active regions, mainly at heights below $1 R_{\odot}$. The acceleration of the SEP can hence occur in small regions in the low corona. But the fast flux tube expansion spreads these field lines over a broad longitude range at the source surface.

This does not mean that energetic electrons escape through the entire cross section of the open flux tube. The type III maps do not constrain the depth of the sources and hence their longitudinal extent. In situ measurements of impulsive SEP events actually suggest that energetic particles propagate only in part of these flux tubes. Using Ulysses measurements, Buttighoffer (1998) showed that type III emitting electron beams propagate in flux tubes with a typical size of some $10^{6} \mathrm{~km}$ near $1 \mathrm{AU}$. This corresponds to a heliocentric width of about $2^{\circ}$ at the source surface, which is much smaller than the longitude range of the open coronal flux tubes inferred from the PFSS computations in the present study. Similarly, Mazur et al. (2000) concluded that MeV ions propagate in flux tubes with a diameter of about $5 \times 10^{6} \mathrm{~km}$ at 1 AU, essentially the same as Buttighoffer's value for the electron propagation channels. Given hat the flux tubes may expand by a factor 10-20 between the low corona and the source surface 
(cf. Fig. 1), these values suggest that their cross sections in the low corona may be as small as (1000-2000) km.

The rapid lateral transport of SEP along the coronal magnetic field does not exclude other forms of particle propagation across the average magnetic field. But field line meandering and diffusive cross-field transport will act on longer time scales - e.g., several hours for relativistic electrons, as discussed in Wibberenz \& Cane (2006). Our selection was restricted to events where the initial solar release of energetic particles was accompanied by type III emission. It excludes events where slow mechanisms of cross-field particle transport would become discernible. But the results of the present study show that cross-field transport is not required for explaining the magnetic connection to solar sources far from the nominal Parker spiral - the quasi-static coronal magnetic field clearly can achieve transport over several tens of degrees in longitude.

\subsection{Open magnetic flux tubes and coronal holes}

The present results are also a further illustration of possible solar wind sources in active regions. Neugebauer et al. (2002) found that active regions and coronal holes are the principal source regions of solar wind around the maximum of solar activity. Wang $\&$ Sheeley (2003) then showed, using PFSS calculations, that the solar wind from active regions comes from highly concentrated magnetic flux in the low corona which rapidly expands at greater altitude (see also Schrijver \& DeRosa 2003). The type III bursts and associated open flux tubes in our events give an illustration of that finding.

A conspicuous difference between these open flux tubes and coronal holes is the density. For example, the thermal electron density in type III burst sources around $200 \mathrm{MHz}$, assuming harmonic plasma emission, is about $10^{8} \mathrm{~cm}^{-3}$. From the bursts occurring near the limb (2002 Oct. 05, 2003 Oct. 04) we infer that this level lies about $0.3 R_{\odot}$ above the photosphere. The density in the flux tubes guiding the type III emitting electrons is hence about an order of magnitude above electron densities in coronal holes inferred at this altitude from white light eclipse observations (Koutchmy 1994; Aschwanden 2004, Fig. 1.20).

\section{Summary and conclusions}

We used two complementary techniques to identify the magnetic flux tubes which guide energetic electrons $(30-500 \mathrm{keV})$ and protons $(5-55 \mathrm{MeV})$ through the corona:

- A potential extrapolation of photospheric magnetic field measurements, using a source surface with radius $2.5 R_{\odot}$.

- Imaging of metre-wave type III emission produced by electron beams of some $\mathrm{keV}$ in the corona.

The requirement to have simultaneously an accurate timing of the initial solar release of the particles detected in situ, welldefined metric-to-kilometric type III bursts observed with time resolution $\leqslant 1 \mathrm{~s}$ at metre waves, and imaging observations at metre wavelengths restricts the sample to 7 SEP events. For these events the two independent techniques provide consistent results:

1. The particles escape from coronal acceleration sites along open magnetic flux tubes whose parent active region is unambiguously identified.

2. The open flux tubes diverge rapidly with height, from a few degrees in longitude at the photospheric level to several tens of degrees at the source surface.
3. These flux tubes connect the parent active region to the Parker spiral at the source surface even when the active region is as far as $50^{\circ}$ away from the nominal connection longitude.

4. The magnetic field polarity of the open field lines in the PFSS extrapolation correlates with the streaming direction of the escaping energetic electrons.

5. In two cases where the inferred coronal flux tubes connect to the source surface at high heliolatitudes, there is evidence that the interplanetary magnetic field lines bend down to the ecliptic before reaching $1 \mathrm{AU}$.

Acknowledgements. Research with Wind/3DP is funded by NASA grant NAG56928 at Berkeley. The Nançay Radio Observatory is funded by the French Ministry of Education, the CNRS and the Région Centre. Part of this research was supported by the France Berkeley Fund. This work benefitted from the data of MDI and CELIAS/MTOF aboard SoHO, a project of international cooperation between ESA and NASA, and from the SWE experiment aboard Wind. Extensive use was made of the PFSS code developed by C.J. Schrijver and M.L. DeRosa at Lockheed Martin Solar and Astrophysics Lab and available under SolarSoft. M.L. DeRosa is acknowledged for his most helpful assistance. The authors are grateful to the generous data supply from the radio spectrographs in Potsdam $(\mathrm{H}$. Aurass), Athens (A. Hilaris), Moscow (I. Chertok), and Nançay (A. Lecacheux). We acknowledge numerous discussions with S. Hoang and with H.S. Hudson who also drew our attention to the PFSS routines in SolarSoft, and very helpful comments by the referee.

\section{References}

Aschwanden, M. J. 2004, Physics of the Solar Corona, An Introduction (Springer \& Praxis Publishing)

Aschwanden, M. J., Bastian, T. S., Benz, A. O., \& Brosius, J. W. 1992, ApJ, 391, 380

Aurass, H., \& Klein, K.-L. 1997, A\&AS, 123, 279

Aurass, H., Klein, K.-L., \& Martens, P. C. H. 1994, Sol. Phys., 155, 203

Benz, A. O. 1993, Plasma astrophysics: Kinetic processes in solar and stellar coronae (Dordrecht: Kluwer), 184

Bougeret, J.-L., Kaiser, M. L., Kellogg, P. J., et al. 1995, Space Sci. Rev., 71, 231

Buttighoffer, A. 1998, A\&A, 335, 295

Carmichael, H. 1962, Space Sci. Rev., 1, 28

Caroubalos, C., Maroulis, D., Patavalis, N., et al. 2001, Exper. Astron., 11, 23

Dulk, G. A., Steinberg, J. L., Hoang, S., \& Lecacheux, A. 1986, in The Sun and the Heliosphere in Three Dimensions, ed. R. G. Marsden, 123, 229

Duncan, R. A. 1979, Sol. Phys., 63, 389

Fan, C. Y., Pick, M., Pyle, R., Simpson, J. A., \& Smith, D. R. 1968, J. Geophys. Res., 73, 1555

Hoang, S., Poquérusse, M., \& Bougeret, J.-L. 1997, Sol. Phys., 172, 307

Hovestadt, D., Hilchenbach, M., Burgi, A., et al. 1995, Sol. Phys., 162, 441

Ippolito, A., Pommois, P., Zimbardo, G., \& Veltri, P. 2005, A\&A, 438, 705

Kallenrode, M.-B., Cliver, E. W., \& Wibberenz, G. 1992, ApJ, 391, 370

Kerdraon, A., \& Delouis, J. 1997, in Coronal Physics from Radio and Space Observations, ed. G. Trottet Lect. Not. Phys. (Springer), 483, 192

Klein, K.-L., \& Posner, A. 2005, A\&A, 438, 1029

Klein, K.-L., Aurass, H., Soru-Escaut, I., \& Kalman, B. 1997, A\&A, 320, 612

Klein, K.-L., Krucker, S., Trottet, G., \& Hoang, S. 2005, A\&A, 431, 1047

Koutchmy, S. 1994, in Adv. Space Res., 14(4), 29

Kundu, M. R., Raulin, J. P., Nitta, N., et al. 1995, ApJ, 447, L135

Kunow, H., Wibberenz, G., Green, G., Müller-Mellin, R., \& Kallenrode, M.-B. 1991, in Physics of the Inner Heliosphere II, ed. R. Schwenn, \& E. Marsch (Springer-Verlag), 21, 243

Lecacheux, A. 2000, in Radio Astronomy at Long Wavelengths, ed. R. Stone, K. Weiler, M. Goldstein, \& J.-L. Bougeret, AGU Monograph, 119, 321

Lin, R. P. 1970, Sol. Phys., 15, 453

Lin, R. P., Anderson, K. A., Ashford, S., et al. 1995, Space Sci. Rev., 71, 125

Lointier, G., Klein, K.-L., \& Hoang, S. 2004, in SF2A-2004: Semaine de l'Astrophysique Francaise, ed. F. Combes, D. Barret, T. Contini, F. Meynadier, \& L. Pagani, 117

Mann, G., Aurass, H., Voigt, W., \& Paschke, J. 1992, in Coronal Streamers, Coronal Loops, and Coronal and Solar Wind Composition, ESA SP, 48, 129 Mazur, J. E., Mason, G. M., Dwyer, J. R., et al. 2000, ApJ, 532, L79 
McLean, D., \& Melrose, D. B. 1985, in Solar Radiophysics: Studies of Emission from the Sun at Metre Wavelengths, ed. D. McLean, \& N. Labrum (Cambridge University Press), 237

Neugebauer, M. 1999, Rev. Geophys., 37, 107

Neugebauer, M., Liewer, P. C., Smith, E. J., Skoug, R. M., \& Zurbuchen, T. H. 2002, J. Geophys. Res., 107, SSH 13

Nitta, N. V., Reames, D. V., DeRosa, M. L., et al. 2006, ApJ, 650, 438

Nolte, J. T., \& Roelof, E. C. 1973, Sol. Phys., 33, 241

Ogilvie, K. W., Chornay, D. J., Fritzenreiter, R. J., et al. 1995, Space Sci. Rev., 71,55

Perez-Peraza, J. 1986, Space Sci. Rev., 44, 91

Pick, M., Mason, G. M., Wang, Y.-M., Tan, C., \& Wang, L. 2006, ApJ, 648, 1247

Raulin, J. P., Kundu, M. R., Hudson, H. S., Nitta, N., \& Raoult, A. 1996, A\&A, 306, 299
Reames, D. V. 1999, Space Sci. Rev., 90, 413

Reinhard, R., \& Wibberenz, G. 1974, Sol. Phys., 36, 473

Scherrer, P. H., Bogart, R. S., Bush, R. I., et al. 1995, Sol. Phys., 162, 129

Schrijver, C. J. 2001, ApJ, 547, 475

Schrijver, C. J., \& DeRosa, M. L. 2003, Sol. Phys., 212, 165

Schrijver, C. J., \& Title, A. M. 2001, ApJ, 551, 1099

Trottet, G., Pick, M., House, L., et al. 1982, A\&A, 111, 306

van Hollebeke, M. A. I., Ma Sung, L. S., \& McDonald, F. B. 1975, Sol. Phys.,

$$
\text { 41, } 189
$$

Wang, Y.-M., \& Sheeley, Jr., N. R. 2003, ApJ, 587, 818

Wang, Y.-M., Pick, M., \& Mason, G. M. 2006, ApJ, 639, 495

Wibberenz, G., \& Cane, H. V. 2006, ApJ, 650, 1199

Zlotnik, E. Y., Klassen, A., Klein, K.-L., Aurass, H., \& Mann, G. 1998, A\&A, 331,1087 